\title{
Single clips versus multi-firing clip device for closure of mucosal incisions after peroral endoscopic myotomy (POEM)
}

Authors

Institution
Tessa Verlaan, Fraukje A. M. Ponds, Barbara A. J. Bastiaansen, Albert J. Bredenoord, Paul Fockens

Department of Gastroenterology and Hepatology, Academic Medical Center, University of Amsterdam, Amsterdam, The Netherlands submitted:

21. February 2016 accepted after revision: 13. June 2016

\section{Bibliography}

DOI http://dx.doi.org/

10.1055/s-0042-113126

Published online: 21.9.2016

Endoscopy International Open

2016; 04: E1052-E1056

(c) Georg Thieme Verlag KC

Stuttgart · New York

E-ISSN 2196-9736

Corresponding author

Paul Fockens, MD PhD

Department of

Gastroenterology and

Hepatology

Academic Medical Center

University of Amsterdam

Meibergdreef 9

1105 AZ Amsterdam

The Netherlands

Fax: +31-20-5664440

p.fockens@amc.uva.nl
Background and aims: After Peroral Endoscopic Myotomy (POEM), the mucosal incision is closed with endoscopically applied clips. After each clip placement, a subsequent clipping device has to be introduced through the working channel. With the Clipmaster3, three consecutive clips can be placed without reloading which could reduce closure time. We performed a prospective study evaluating efficacy, safety, and ease of use. Closure using Clipmaster3 was compared to closure with standard clips.

Methods: Patients undergoing closure with the Clipmaster3 were compared to patients who underwent POEM with standard clip closure.

Results: In total, 12 consecutive POEM closures with Clipmaster3 were compared to 24 standard POEM procedures. The Clipmaster3 and the standard group did not differ in sex distribution, age (42 years [29-49] vs 41 years [34-54] $P=$ 0.379 ), achalasia subtype, disease duration,

\section{Introduction}

\section{$\nabla$}

Peroral Endoscopic Myotomy (POEM) was introduced in 2010 as an alternative endoscopic treatment for achalasia [1]. The technique is suggested to combine the benefits of the current standard treatment options for achalasia, the minimally invasive approach of endoscopic pneumodilation with the efficacy of laparoscopic Heller myotomy. The POEM procedure is performed entirely endoscopically and starts with a longitudinal incision of the mucosa allowing entry to the submucosa. The endoscope is then advanced into the submucosa to create a submucosal tunnel. After a myotomy of the circular muscle fibers within the submucosal tunnel, the entry site of the submucosal tunnel is closed with endoscopic clips approximating the mucosa again.

At expert centers worldwide, more than 4000 achalasia patients underwent a POEM procedure for achalasia over the last 5 years. Initial outcomes length of the mucosal incision $(25.0 \mathrm{~mm}$ [20 - 30] vs $20.0 \mathrm{~mm}$ [20-30], $P=1.0$ ), and closure time (622 seconds [438-909] vs 599 seconds [488664] $P=0.72$ ). Endoscopically successful closure could be performed in all patients. The proportion of all clips used that were either displaced or discarded was larger for Clipmaster3 (8.8\%) compared to standard closure $(2.0 \%, P=0.00782)$. Ease of handling VAS (visual analogue scale) score for Clipmaster3 did not differ between endoscopist and endoscopy nurse ( 7 out of 10 ).

Conclusions: Clipmaster3 is feasible and safe for closure of mucosal incisions after POEM. Clipmaster3 was not associated with reduced closure time. Compared to standard closure, more Clipmaster3 clips were displaced or discarded to achieve successful closure. A training effect cannot be excluded as a cause of these results.

Study registration: NCT01405417

for POEM concerning safety and efficacy are encouraging. Short-term clinical success ranges from $82 \%$ to $100 \%[2,3]$.

Safety and clinical success of POEM are based on a careful execution of the subsequent steps of which the procedure consists. Performing POEM is time consuming. Mean procedure time of available studies approximates 120 minutes [2]. A recent publication showed a learning curve reducing procedure times to a median of 97 [65-140] minutes after 93 patients [4]. As more experience is gained with performing POEM, modifying the procedure could further improve the outcomes of efficacy and safety. Moreover, the procedure could be shortened by optimizing the different phases of the procedure. For closure of the mucosal entrance, endoscopic metal clips are often the method of choice because endoscopists are experienced in using these clips. After placement of each clip, a clipping device then has to be introduced through the working channel of the endo- 
scope. Recently, a new endoscopic clip system has been introduced, the Clipmaster3. The Clipmaster 3 is based on the standard endoscopic clip but is able to place up to three consecutive clips without having to reload clips after positioning. Using this clip system, closure time of the mucosal entry site could potentially be reduced and this may help to shorten the total POEM procedure time. Moreover, we hypothesize that Clipmaster3 is as safe and effective as standard clip closure for POEM. In this study, we prospectively evaluated closure time of the Clipmaster3, its safety, and ease of use. Moreover, closure using Clipmaster3 was compared to closure with standard endoscopic clips.

\section{Methods}

\section{$\nabla$}

Patients diagnosed with achalasia based on high resolution manometry and timed barium esophagram who underwent a POEM between 2011 and 2014 were prospectively entered in a database at the Academic Medical Center, Amsterdam, The Netherlands. From April 2013 until March 2014, in consecutive achalasia patients who underwent POEM, the Clipmaster3 was used for closure of the mucosal incision after the myotomy was completed. Selection of patients was based on logistics, the same team of endoscopy nurse and endoscopist performing all of the studied POEM procedures, as well as the availability of the Clipmaster3 system. As a result, for 12 consecutive procedures, Clipmaster3 was planned to be used for the mucosal closure during POEM. The Clipmaster3 is a clip applicator in which three clips are preloaded. Patients undergoing closure with the Clipmaster3 were compared to a cohort of achalasia patients who underwent POEM in which standard endoscopic clips were used for closure. Before the first procedure, both endoscopist and endoscopy nurse were trained on an ex-vivo model to use Clipmaster3 by placing 30 clips. Data for the selected patients were collected by the same person for all procedures in a prospective manner or during the procedure. Closure time was also checked after the procedure for each patient with the video taken during the procedure.

\section{POEM procedure}

The POEM procedure was executed by an experienced gastroenterologist and performed according to the previously described procedure [5]. In short, the following steps can be distinguished: (1) incision of the mucosa to create an entrance for the submucosal tunnel; (2) creation of a submucosal tunnel $10-14 \mathrm{~cm}$ proximally to the lower esophageal sphincter to a maximum of $3 \mathrm{~cm}$ into the cardia of the stomach; (3) myotomy of the circular muscle fibers starting $2 \mathrm{~cm}$ distally from the lower end of the incision and into the stomach; (4) closure of the mucosal entry site of the submucosal tunnel using the Clipmaster3 system or standard endoscopic clips.

\section{Mucosal closure using standard endoscopic clips}

Endoscopic metal clips (HX-610-135L Single Use Clips, Olympus) were used with a rotatable Clip Reusable Rotatable Clip Fixing Device (HX-110UR EZ, Olympus). The width of the open clip is $9 \mathrm{~mm}$, the length of each arm of the clip is $5 \mathrm{~mm}$, and the clip is rotatable over 360 degrees. After placement of each clip, the device was removed from the endoscope, reloaded with a new clip, and reintroduced through the working channel of the endoscope.
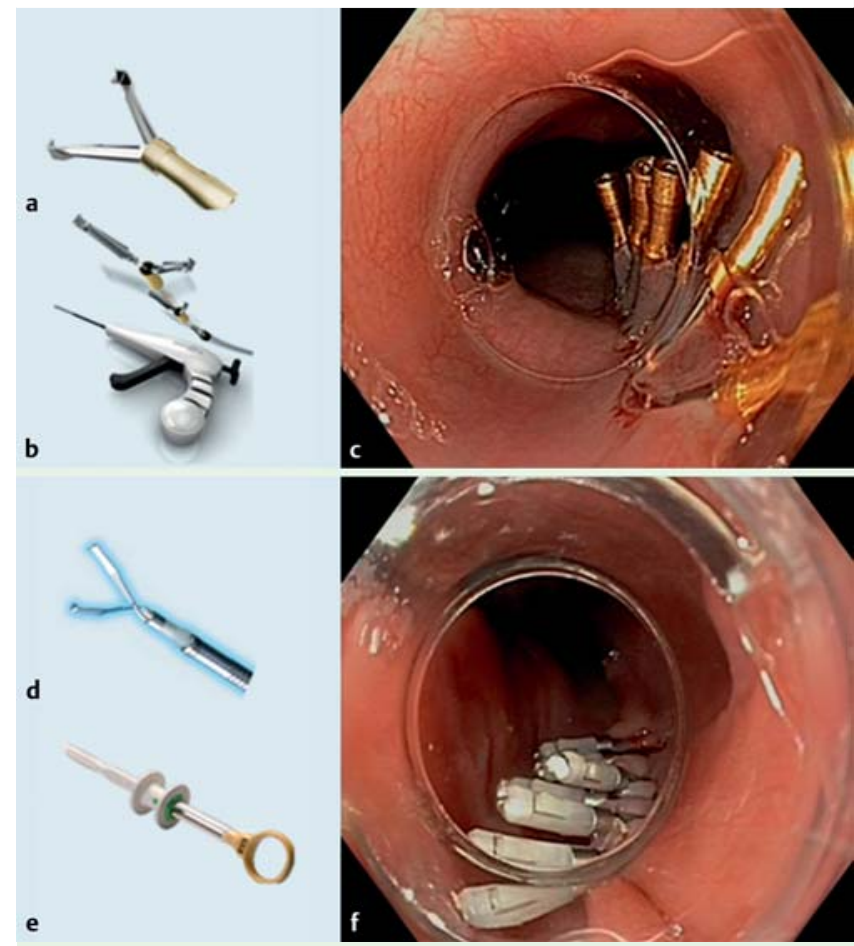

Fig. 1 Clipmaster3 clip versus standard endoscopic clip. a Clipmaster3 clip. b Clipmaster3 device. c Closed mucosal incision with Clipmaster3. d Olympus clip. e Rotatable Clip Delivery device. f Closed mucosal incision with Olympus clips (@ 2016 olympus europa SE \& Co. UG).

\section{Mucosal closure using Clipmaster 3 system}

The Clipmaster3 (SPE1-X3 - 26-220-502063, Medwork, GmbH, Aisch, Germany) is a clip system with three clips preloaded. As a result, up to three clips can be placed without reloading. The clip applicator has a diameter of $2.6 \mathrm{~mm}$, the width of the open clip is $12 \mathrm{~mm}$, and the clip is rotatable over 360 degrees. The clips have small hooks attached to its jaws for mucosal approximation. After usage of the three clips, the device was removed after which a new device was introduced containing again three clips (๑ Fig.1).

\section{Outcome parameters}

The following variables were collected for both groups: sex, age, achalasia subtype based on high-resolution manometry, preoperative esophageal diameter based on soluble-contrast X-swallow, disease duration, previous achalasia treatment, medication use, length of the mucosal incision, procedure time, endoscopically successful closure, leakage on X-swallow 1 day post-procedure, closure time, number of clips used for successful closure, total number of clips, unsuccessfully placed clips, adverse events, postoperative stay, readmission within 30 days, and mortality. The definition of successful endoscopic closure was stated as the absence of submucosal leakage of water-soluble contrast visible during the X-swallow 1 day post-procedure. Closure time was defined as the time interval between the first appearance of a clip in the esophageal lumen until placement of the last clip. Adverse events were defined as any deviation from the normal postoperative course within 30 days after POEM. Moreover, handling of the Clipmaster3 was evaluated by means of a questionnaire where ease of use was scored on a VAS (visual analogue scale, $0=$ impossible, $10=$ very easy) by both endoscopist as well as the endoscopy nurse assisting with the closure procedure. Pos- 
sible underlying causes related to a difficult closure of the submucosal entrance by the Clipmaster3 were also noted on the questionnaire.

\section{Data analysis}

In total, 12 consecutive POEM closures with Clipmaster3 were compared to 24 consecutive standard procedures. The same physician and endoscopy nurse performed all procedures. This hampered a case-match analysis and data were compared in a 1:2 manner.

\section{Results}

$\nabla$

Between 2013 and 2014, 12 patients underwent a POEM procedure in which the mucosal entry site was closed with Clipmaster3. From August 2011 to 2014, a total of 71 patients underwent standard POEM. Of all patients who underwent standard POEM, 24 patients underwent a POEM procedure by the same team of endoscopist and endoscopy nurse.

The Clipmaster3 and the standard group did not differ in sex distribution, age at surgery (42 years [29-49] vs 41 years [34-54] $P$ $=0.379)$, diagnosed type of achalasia $(P=0.181)$, preoperative esophageal diameter based on soluble-contrast X-swallow (27 $\mathrm{mm}$ [23-37] vs $27 \mathrm{~mm}$ [22-35], $P=0.724)$, disease duration (57.0 months [39.3-121.3] vs 38.0 months [18.0-97.8], $P=$ $0.137)$, length of the mucosal incision ( $25.0 \mathrm{~mm}$ [20-30] vs 20.0 mm [20-30], $P=1.0$ ), or medication use ( Table 1 ). More patients underwent previous therapy before POEM in the Clipmaster3 group (11 out of 12 (91.7\%) vs 19 out of 24 (79.2\%), $P=$ 0.020).

Endoscopically successful closure without mucosal gaps could be performed in both groups in all patients. In none of the patients was leakage seen during water soluble-contrast X-swallow on the morning after the procedure. Duration of closure time of the mucosal incision did not differ between the groups (622 seconds [438 - 909] vs 599 seconds [488-664], $P=0.72$ ) ( $\bullet$ Table 2). Total procedure time did not differ between the groups (95 min [81112] vs $98 \min [84.3-120] P=0.909$ ).
The number of clips that were placed to achieve successful closure did not differ between groups (8 [7-9] vs 8 [7-9], $P=$ 0.902) ( Table 2). For Clipmaster3 compared to standard closure, a larger proportion of all clips used were either misplaced or discarded ( $8.8 \%$ vs $2.0 \%, P=0.00782$ ). The number of adverse events and postoperative stay did not differ between groups. There was no mortality and there were no readmissions within 30 days in either group.VAS score for the ease of use for handling of the Clipmaster3 for closure did not differ between the endoscopist and endoscopy nurse and were both 7 ([6-9], [6-8]) out of 10 (Table 3). Reasons for difficult closure for the endoscopist were type of incision and the anatomy of the esophagus, and for the endoscopy nurse, reasons for difficult placement were unpredictable opening and handling of the clip ( Table 3 ).

\section{Discussion}

In this study, we prospectively evaluated safety and efficacy, closure time, as well as ease of use of the new Clipmaster3 system for endoscopic closure of the mucosal incision after POEM. Clipmaster3 proved to be feasible and safe for mucosal closure after POEM in the 12 patients studied. In contrast to our hypothesis, no reduction in closure time was observed for Clipmaster3 compared to standard clip closure. More clips from Clipmaster3 were misplaced during closure compared to the standard clips. Handling the Clipmaster3 system was independently evaluated as moderately easy by both endoscopist and endoscopy nurse and was scored as 7 out of 10 on a VAS. The patients studied were compared based on the same trained endoscopy nurse attending all procedures as well as the same physician performing all POEM procedures.

In the majority of publications on POEM, endoclips are used for closure of the mucosal incision. An alternative closure modality has been described in only one small study. Meireles et al. used surgical tissue glue to complete closure in addition to standard clips in five patients [6]. However, their study showed that closure with endoclips alone was already sufficient for effective mucosal closure. Several cases of rescue closure attempts for compli-

\begin{tabular}{|c|c|c|c|c|}
\hline & $\begin{array}{l}\text { Clipmaster3 } \\
(n=12)\end{array}$ & $\begin{array}{l}\text { Standard } \\
(\mathrm{n}=\mathbf{2 4 )}\end{array}$ & $P$ value & Table 1 Patient demographics. \\
\hline Female gender, $\mathrm{n}(\%)$ & $7(58.3)$ & $12(50)$ & 0.64 & \\
\hline Age at surgery, median [range], years & $42[29-49]$ & $41[34-54]$ & 0.38 & \\
\hline Achalasia type, $\mathrm{n}(\%)$ & & & 0.18 & \\
\hline Type I & $5(41.7)$ & $7(2.9)$ & & \\
\hline Type II & $6(50)$ & $12(50)$ & & \\
\hline Type III & $0(0)$ & $5(2.0)$ & & \\
\hline Unknown & $1(0.8)$ & $0(0)$ & & \\
\hline Disease duration, median [interquartile range], months & $57.0[39.3-121.3]$ & $38[18.0-97.8]$ & 0.14 & \\
\hline Previous therapy, $\mathrm{n}(\%)$ & $11(91.7)$ & $19(79.2)$ & 0.02 & \\
\hline Botox & $1(9.0)$ & $1(5.3)$ & & \\
\hline Pneumodilation & $9(81.8)$ & $6(31.6)$ & & \\
\hline Heller myotomy & $1(9.0)$ & $12(63.2)$ & & \\
\hline Medication at time of surgery, $\mathrm{n}(\%)$ & 0 & $4(16.7)$ & 0.59 & \\
\hline NSAIDs & 0 & 1 & & \\
\hline Anti-platelets & 0 & 2 & & \\
\hline Corticosteroids & 0 & 1 & & \\
\hline $\begin{array}{l}\text { Esophageal diameter based on soluble-contrast X-swallow, } \\
\text { median [interquartile range], mm }\end{array}$ & $27[23-37]$ & $27[22-35]$ & 0.72 & \\
\hline Length of incision, median [interquartile range], mm & $25.0[20-30]$ & $20.0[20-30]$ & 1.00 & \\
\hline
\end{tabular}

Esophageal diameter was measured during soluble-contrast X-swallow before POEM. 


\begin{tabular}{|c|c|c|c|}
\hline & $\begin{array}{l}\text { Clipmaster3 } \\
(n=12)\end{array}$ & $\begin{array}{l}\text { Standard } \\
(\mathrm{n}=24)\end{array}$ & $P$ value \\
\hline Procedure time, median [interquartile range], minutes & $95[81-112]^{*}$ & $98[84.3-120]$ & 0.90 \\
\hline Successful macroscopic closure, n (\%) & $12(100)$ & $24(100)$ & \\
\hline Closure time, median [interquartile range], seconds & $622[438-909]$ & $599[488-664]$ & 0.72 \\
\hline $\begin{array}{l}\text { Number of clips for successful closure, median } \\
\text { [interquartile range], } \mathrm{n}\end{array}$ & $8[7-9]$ & $8[7-9]$ & 0.90 \\
\hline Total number of clips used, $\mathrm{n}$ & 102 & 191 & \\
\hline Number of unsuccessful clips, $n$ (\% of all clips) & $9(8.8)$ & $4(2.0)$ & 0.00 \\
\hline Displaced clips & 6 & 3 & \\
\hline Discarded clips & 3 & 1 & \\
\hline Adverse events, $\mathrm{n}(\%)$ & $3(25)$ & $6(25)$ & 1.00 \\
\hline Nausea & 1 & 1 & \\
\hline Fever & 1 & 1 & \\
\hline Reflux & 1 & 1 & \\
\hline Bleeding & 0 & 1 & \\
\hline Allergic reaction & 0 & 1 & \\
\hline Hypotension & 0 & 1 & \\
\hline Postoperative hospital stay, $\mathrm{n}$ [range] & $1[1-1]$ & $1[1-2]$ & \\
\hline Readmission within 30 days, $\mathrm{n}$ & 0 & 0 & \\
\hline Mortality, n & 0 & 0 & \\
\hline
\end{tabular}

* One missing value.

\begin{tabular}{|c|c|c|}
\hline & \multicolumn{2}{|c|}{$\begin{array}{l}\text { Clipmaster3 } \\
(n=12)\end{array}$} \\
\hline & Nurse & Endoscopist \\
\hline Ease of use $(0-10)$, median [interquartile range] & $7.0[6-9]$ & $7.0[6-8]$ \\
\hline Reasons for difficult closure, $\mathrm{n}(\%)$ & $6(50)$ & $4(33.3)$ \\
\hline Anatomy of incision & 0 & 1 \\
\hline Difficult positioning & 0 & 1 \\
\hline Wide esophagus & 0 & 1 \\
\hline Unreliable & 1 & 1 \\
\hline Difficult detachment of clip & 1 & 0 \\
\hline Difficult handling & 2 & 0 \\
\hline Clip does not open enough & 1 & 0 \\
\hline Difficult removal of clips & 1 & 0 \\
\hline
\end{tabular}

Table 3 Ease of use of Clipmaster 3 based on VAS scale.

Ease of use is scored via a VAS (visual analogue scale) defined as $0=$ impossible, $10=$ very easy.

cated POEM procedures with modalities other than standard clips have been described. Endoscopic suturing (Overstitch Endoscopic Suturing System; Apollo Endosurgery, Austin, TX, United States) could be used successfully for closure of the mucosal entry site during a procedure that was complicated by a full thickness muscular rupture [7]. In two other studies, the Over-the-Scope clip was used for closure of a difficult, time consuming case after an initial attempt at closure with numerous endoclips, and this resulted in successful closure [8]. For a perforation during a POEM procedure, fibrin sealant was used which was sprayed into the distal end of the submucosal tunnel and a gastric mucosal penetration was sealed successfully [9]. Moreover, after a failed attempt at closure with metallic clips due to swollen mucosa, tightening of an Endoloop that was snared around the previously placed distal clips resulted in closure of the mucosal incision (the so-called tulip bundle technique) [10].

This is the first study to analyze closure time and investigate improvements in this part of the procedure. Procedure time is mentioned in the majority of the studies, however, closure time is usually not described separately. Procedure time is predominantly determined by creation of the submucosal tunnel which is a time consuming phase of the POEM procedure. However, we are not aware of significant alternative techniques for submucosal tunneling that have been reported in the literature. Moreover, this is the first study that evaluates an alternative closure modality as the initial closure technique and compares it to the established technique.

It was hypothesized that, without subsequent loading of each new clip and without each introduction into the esophagus, closure time could be diminished. However, duration of closure did not differ between standard endoclip closure and closure with the Clipmaster3. It is possible that closure time is not only affected by the subsequent loading and introduction needed before placement of each new clip, but other factors might play a role as well. One explanation could be that the time that was saved by not exchanging devices was invested in clip placement with the slightly more bulky multi-firing device. In the Clipmaster3 group, more clips were misplaced which could have determined closure time more than the time needed to reload each endoclip. Moreover, both endoscopist and endoscopy nurse had less experience with using Clipmaster3 compared to endoclip despite the fact that they had been trained specifically with this device. The above mentioned reasons could have resulted in more clips that failed during placement and resulted in Clipmaster3 being 
more time consuming. In general for POEM, we learned that the key factor for successful closure seems mainly determined by whether the first and second clips are adequately placed. Placement of the first clip just distally to the incision allows the wound edges to align parallel and angle up into the lumen of the esophagus making subsequent clipping easier. Less experience with the Clipmaster 3 could have meant less adequate placement of the first clip and therefore result in more clips being either displaced or discarded. Furthermore, as we gained experience in performing POEM, we observed that the esophageal diameter seemed to influence the level of difficulty of closure as well. We observed that a larger diameter due to long-standing achalasia causes stretching of the mucosa with a wider deviation of the wound edges as a result. Easy and fast approximation of tissue and thus closure could therefore be hampered. However, as measured during X-swallow, the groups did not differ in esophageal diameter before the procedure. Therefore, in this study, this parameter would not have determined closure.

Ease of use of Clipmaster3 was scored as 7 out of 10 for both endoscopist as well as endoscopy nurse, compatible with, on average, moderately easy closure. However, reasons for difficult placement differed between the endoscopist and nurse. For the endoscopy nurse, reasons for difficult placement were unpredictable opening and handling of the clip. For the endoscopist, the type of incision and the anatomy of the esophagus determined difficult closure with Clipmaster3.

Limitations of this study include the small number of patients studied. Moreover, the study was not designed as a randomized controlled trial.

In the authors' opinion, an ideal closure modality for closing the mucosal entry site after POEM should have the ability to rotate easily inside the esophageal space and have a confined opening width to ensure complete grasping of mucosal edges and quick closure. Moreover, the closure modality also determines total costs and therefore should be low cost. In total, standard (Olympus) clips and the fixing device cost around 800 euro per procedure (clipping device 700 euro+10 euro per clip). For Clipmaster3, this sum approximates 450 euro per procedure. The delivery device can however be sterilized and used in multiple patients. This study demonstrates that Clipmaster3 for closure of the mucosal entrance during POEM is as safe and effective for endoscopic closure of a mucosal incision after POEM as standard clips. However, compared to standard metal clips, Clipmaster 3 was not associated with reduced closure time as was hypothesized. Possible reasons for failure of our hypothesis could be associated with the Clipmaster3 being a more voluminous clip system compared to standard clips. Moreover, closure with Clipmaster3 resulted in more dislocated clips and the endoscopy team had less experience with using Clipmaster3 compared to the standard clips.

Competing interests: Paul Fockens: Consultancy Olympus, Covidien, Fujifilm, Cook

\section{Acknowledgments \\ $\nabla$}

All authors disclosed no financial relationships relevant to this publication. TV and FAP contributed to this work by designing the study, extracting and analyzing the data, and drafting the manuscript. $\mathrm{PF}, \mathrm{BB}$, and $\mathrm{AB}$ contributed to the conception and design of the study and critically revised the manuscript. PF performed all study procedures. All authors read and approved the final manuscript.

\section{References}

1 Inoue H, Minami H, Kobayashi $Y$ et al. Peroral endoscopic myotomy (POEM) for esophageal achalasia. Endoscopy 2010; 42: 265 - 271

2 NOSCAR POEM White Paper Committee. Stavropoulos SN, Desilets DJ, Fuchs $\mathrm{KH}$ et al. Per-oral endoscopic myotomy white paper summary. Gastrointest Endosc 2014; 80: 1-15

3 Li QL, Zhou PH. Perspective on peroral endoscopic myotomy for achalasia: Zhongshan experience. Gut Liver 2015; 9: 152-158

4 Patel KS, Calixte R, Modayil RJ et al. The light at the end of the tunnel: a single-operator learning curve analysis for per oral endoscopic myotomy. Gastrointest Endosc 2015; 81: $1181-1187$

5 Verlaan T, Rohof WO, Bredenoord AJ et al. Effect of peroral endoscopic myotomy on esophagogastric junction physiology in patients with achalasia. Gastrointest Endosc 2013; 78: 39-44

6 Meireles OR, Horgan S, Jacobsen GR et al. Transesophageal endoscopic myotomy (TEEM) for the treatment of achalasia: the United States human experience. Surg Endosc 2013; 27: 1803-1809

7 Kurian AA, Bhayani NH, Reavis K et al. Endoscopic suture repair of full thickness esophagotomy during per-oral esophageal myotomy for achalasia. Surg Endosc 2013; 27: 3910

8 Saxena P, Chavez YH, Kord Valeshabad A et al. An alternative method for mucosal flap closure during peroral endoscopic myotomy using an over-the-scope clipping device. Endoscopy 2013; 45: 579-581

9 Li H, Linghu E, Wang X. Fibrin sealant for closure of mucosal penetration at the cardia during peroral endoscopic myotomy (POEM). Endoscopy 2012; 44: 02 UCTN E215 - E216

10 Zhang $Y$, Wang $X$, Fan Z. Reclosure of ruptured incision after peroral endoscopic myotomy using endoloops and metallic clips. Dig Endosc 2014; 26: 295 